\title{
Determination of perfluorooctanesulfonate and perfluorooctanoic acid in sewage sludge samples using liquid chromatography/quadrupole time-of-flight mass spectrometry
}

\author{
Rui Guo, Qunfang Zhou, Yaqi Cai, Guibin Jiang* \\ State Key Laboratory of Environmental Chemistry and Ecotoxicology, Research Center for Eco-Environmental Sciences, \\ Chinese Academy of Sciences, Beijing 100085, China
}

Received 7 November 2007; received in revised form 22 January 2008; accepted 25 January 2008

Available online 3 February 2008

\begin{abstract}
A new method is developed for the determination of perfluorooctanesulfonate (PFOS) and perfluorooctanoic acid (PFOA) in sewage sludge samples. The analytes in sewage sludge samples are extracted by methanol and formic acid, cleaned by C18 solid-phase extraction, then separated, identified and quantitated by liquid chromatography/quadrupole time-of-flight mass spectrometry (LC-QTOF-MS). A C18 column $(150 \mathrm{~mm} \times 2.1 \mathrm{~mm}, 3.5 \mu \mathrm{m})$ with gradient elution of $\mathrm{MeOH}-\mathrm{H}_{2} \mathrm{O}(60: 40)$ containing $5 \mathrm{mmol} / \mathrm{L}$ ammonium acetate and $\mathrm{MeOH}-\mathrm{H}_{2} \mathrm{O}(80: 20)$ is used for the chromatographic separation. $[\mathrm{M}-\mathrm{K}]^{-}$ions at $\mathrm{m} / \mathrm{z} 498.93$ for PFOS and $[\mathrm{M}-\mathrm{COOH}]^{-}$ion at $\mathrm{m} / z 368.97 \mathrm{for}$ PFOA are selected for QTOF-MS in the negative electrospray ionization mode. The detection limits for PFOS and PFOA in sewage sludge samples are 0.5 and $0.8 \mathrm{ng} / \mathrm{g}$, respectively. The spiked recoveries are in the range of 85-114 and 71-98\% for PFOS and PFOA, respectively. The proposed method is successfully applied to the analysis of PFOS and PFOA in 16 sewage sludge samples from China. PFOS and PFOA are detected in most sewage sludge samples and the concentrations of PFOS and PFOA are up to 5383 and $4780 \mathrm{ng} / \mathrm{g}$ (oven dry weight), respectively.

(C) 2008 Elsevier B.V. All rights reserved.
\end{abstract}

Keywords: PFOS; PFOA; Sewage sludge; HPLC-QTOF

\section{Introduction}

Perfluorinated surfactants perfluorooctanesulfonate (PFOS) and perfluorooctanoic acid (PFOA) are additives in a wide variety of industrial products and commodities, including protective coatings for carpets and apparel, paper coatings, insecticide formulations, and surfactants [1]. PFOS is also used as a surfactant in firefighting foams [2]. It spreads widely in the environment, wildlife, and humans [3-10]. Subchronic exposure to PFOS may lead to significant weight loss accompanied by hepatotoxicity and reductions of serum cholesterol and thyroid hormones. It has also been reported that PFOA can produce hepatotoxicity, anorexia, alteration of fatty acid metabolism, reduction of circulating thyroid hormones and androgen, bradycardia, and hypothermia in the rat [11]. The 3M Company, one of the most fluorochemical manufacturing companies, has claimed to cease

\footnotetext{
* Corresponding author. Tel.: +86 1062849179 ; fax: +86 1062849179.

E-mail address: gbjiang@rcees.ac.cn (G. Jiang).
}

the manufacturing of perfluorooctanyl-related materials in 2000 . Products made of perfluorooctanyl-related materials, however, are still widely available all over the world. Some publications have demonstrated widespread distribution of PFCs in China [12]. The distribution mode and the contamination levels of PFCs in some matrices such as sewage sludge are still not clear now and worthy of being studied in China.

High-performance liquid chromatography (HPLC) with triple quadrupole mass spectrometry in electrospray negative mode is the most extensively applied method for the analysis of PFCs in various environmental and biological matrices, because triple quadrupole mass spectrometry has high precision, wide linear range and high sensitivity for PFOS and PFOA determination in complicated matrices. It is able to determinate specific fragmentation of isolated precursor ions and eliminate background noise. More and more laboratories have HPLC coupled with triple quadrupole mass spectrometry, however, most of them have no ability to analysis PFOS and PFOA due to the high instrument blank. So auxiliary line fittings of LC/MS/MS should be stainless to avoid the contamination, which greatly 
increases the experimental cost. Now only a few laboratories can afford it. The application of HPLC-MS in the selected ion monitoring (SIM) mode and HPLC-ion trap mass spectrometry has recently been reported as the attractive alternatives for the analysis of perfluorooctanesulfonate and related fluorochemicals [13-15]. However, when these methods are applied to detect low concentrations of PFCs in complicated matrix, the interference of matrix must be carefully gotten rid of. Other mass spectrometric techniques such as quadrupole time-of-flight mass spectrometry (QTOF-MS) are also applied in the environmental samples analysis for structure elucidation or confirmation purposes because it has high resolution and sensitivity. QTOFMS has distinct advantages over other scanning instruments including the detection of a high percentage of ions, high mass resolution and accuracy, fast acquisition rates, high sensitivity, and large mass range. QTOF-MS technique is considered to be one of the attractive alterations for the unequivocal identification of unknown compounds using full-spectrum scan. However, it is seldom applied to quantitative analysis because of its relatively narrow linear range for most of the compounds. Recently, several papers have reported about liquid chromatography/quadrupole time-of-flight mass spectrometry (LC-QTOF-MS) being used to detect trace levels of organic pollutions [16,17]. It not only can record selected precursor ions, but also can offer high mass resolution and accuracy. Considering its specific advantage of high mass accuracy and sensitivity, we apply it to determine PFOS and PFOA in sewage sludge samples as there is no report on the application of LC-QTOF-MS in the analysis of PFCs in environmental samples. PFOS and PFOA can be confirmed by the retention time and the accurate mass spectrometry. The single MS mode is used for both qualitative and quantitative analysis. The MS/MS mode can be applied to identify PFOS and PFOA in more detail. The proposed method is applied to accurate and precise analysis of PFOS and PFOA in 16 sewage sludge samples.

\section{Experimental}

\subsection{Chemicals and reagents}

Potassium PFOS was purchased from Fluka (Milwaukee, MI, USA). PFOA and perfluoroheptanoic acid (PFHpA, as lock mass standard) were purchased from Aldrich (Milwaukee, MI, USA). HPLC-grade methanol was purchased from J.T. Baker (Philipsburg, NJ, USA). Ultra-pure water was prepared by MilliQ system (Millipore, Milford, MA, USA). Ammonium acetate $\left[\mathrm{CH}_{3} \mathrm{COONH}_{4}\right]$ and acetic acid $\left(\mathrm{CH}_{3} \mathrm{COOH}\right)$ were of analytical reagent grade. The $\mathrm{C} 18 \mathrm{SPE}$ cartridge $(500 \mathrm{mg}, 6 \mathrm{~mL})$ was bought from Alltech (Deerfield, IL, USA).

The nitrogen gas $\left(\mathrm{N}_{2}\right)$ was used as evaporation and nebulization gas. The argon was used as collision gas. All stock solutions were kept in polypropylene volumetric flasks at $4{ }^{\circ} \mathrm{C}$ in the dark.

\subsection{Instrumentation}

The analysis of PFOS and PFOA are carried out by the hyphenated system of HPLC with quadrupole time-of-flight mass spectrometry. A model Alliance 2695 from Waters (Waters Corp., USA) is used to separate PFOS and PFOA. The separate column is XTerra C18 column (particle size of $3.5 \mu \mathrm{m}, 2.1 \mathrm{~mm}$ i.d. $\times 150 \mathrm{~mm}$ in length, Waters, Ireland) and the guard column is XTerra C18 $2.1 \mathrm{~mm} \times 10 \mathrm{~mm}$ packed with particle size of $3.5 \mu \mathrm{m}$ (Waters, Ireland). Optimum separation is achieved with a binary mobile phase at a flow rate of $300 \mu \mathrm{L} / \mathrm{min}$. A deionized water solution containing $5 \mathrm{mmol} / \mathrm{L}$ ammonium acetate $(\mathrm{pH}$ 6.0) and methanol serve as mobile phase. According to previous studies, volatile buffer ammonium acetate in the mobile phase is essential to the ionization of PFOA and PFOS $[18,19]$ because its suppressing effect on the signal is weak. Additionally, as a buffer compound, it is effective at $\mathrm{pH}$ 6.0, which is found to be the optimum value of $\mathrm{pH}$ for the separation of PFCs. The gradient starts at $60 \%$ methanol followed by $0.5 \mathrm{~min}$ ramp to $80 \%$ methanol, hold $9 \mathrm{~min}$, and then reverting to initial conditions by $0.5 \mathrm{~min}$ ramp allowing $8 \mathrm{~min}$ stabilization time.

A quadrupole time-of-flight (QTOF micro) mass spectrometer (Micromass, Manchester, UK) with a Z-spray ESI source working in negative mode is used for the identification and quantitative analysis of PFOS and PFOA. Optimal ionization source working parameters are as follows: capillary and sample cone voltages, $2.5 \mathrm{kV}$ and $35 \mathrm{~V}$; source and desolvation temperatures, 120 and $250^{\circ} \mathrm{C}$; cone and desolvation gas flows (nitrogen), 100 and $650 \mathrm{~L} / \mathrm{h}$, respectively. The collision gas is argon at the pressure of $5.0 \times 10^{-5}$ Torr and the collision energy is $35 \mathrm{~V}$. Both the high- and low-resolution for mass filter are set at $10.0 \mathrm{~V}$. The pressure in the TOF cell is lower than $5.0 \times 10^{-7}$ Torr $(1$ Torr $=133.322 \mathrm{~Pa}) . \mathrm{m} / \mathrm{z} 498.93 \pm 0.05$ for PFOS and $[\mathrm{M}-\mathrm{COOH}]^{-}$ion at $\mathrm{m} / z 368.97 \pm 0.05$ for PFOA are monitored for their accurate identification and the selection of precursor ions.

The TOF analyzer is calibrated every day for the accurate mass analysis. The lock mass is utilized to guarantee the mass accuracy. Continuum mode TOF mass spectra are recorded using single MS modes from $\mathrm{m} / \mathrm{z} 50$ to 600 with a duty cycle of $1.0 \mathrm{~s}$. The acquired data are converted to centroid (80\% of the top peak area) to implement lock mass adjustment and to generate the accurate mass spectra. A window width of $\pm 0.05 \mathrm{Da}$ is selected to obtain enough selectivity and to decrease the noise (i.e. better limits of detection). For MS/MS mode, the precursor ion is selected in the quadrupole analyzer and fragmented in the hexapole cell by applying collision cell offset voltages $35 \mathrm{~V}$, depending on the compound. Product ion spectra are recorded in the TOF analyzer from $\mathrm{m} / \mathrm{z} 50$ to 600 , too. Afterwards, the spectra are lock mass corrected using the $\mathrm{m} / \mathrm{z}$ value of the precursor ion. Data are collected and processed by MassLynx v4.1 software.

\subsection{Sample collection}

Sewage sludge samples were collected in polypropylene bottles from 16 municipal waste water treatment plants in February 2004. They were transported in ice and frozen $\left(-15^{\circ} \mathrm{C}\right)$ until analysis. The sewage sludge samples were firstly grounded and homogenized in a methanol-rinsed carnelian mortar. Each sewage sludge sample was dried in oven at $103^{\circ} \mathrm{C}$ overnight 
to eliminate aliquots. The concentration of PFOS and PFOA in sewage sludge samples were calculated and expressed in the unit of ng/g (oven dry weight).

\subsection{Sample preparation}

A simple and efficient sample pretreatment method including extraction and cleanup steps used herein is similar to that described elsewhere [20], which offers satisfying quantitative recoveries of anionic PFCs in sewage sludge matrix. Briefly, approximate $0.1 \mathrm{~g}$ of oven dried sewage sludge samples are extracted by the mixture of $1 \%$ formic acid and methanol. In order to enrich the analytes and to remove the acetic acid, salts, and potential matrix interferences, the combined sludge extract is then loaded at a flow rate of $1-2 \mathrm{~mL} / \mathrm{min}$ on a vacuum manifold onto a 500-mg C18 SPE cartridges which has been pre-conditioned with $10 \mathrm{~mL}$ of methanol followed by $10 \mathrm{~mL}$ of $1 \%$ acetic acid. The SPE cartridges are then rinsed with $10 \mathrm{~mL}$ of Milli-Q water, dried under vacuum for at least $2 \mathrm{~h}$. PFCs are eluted from the cartridge with $4 \mathrm{~mL}$ of methanol and collected in $1: 1(\mathrm{v} / \mathrm{v})$ methanol/acetone-washed graduated glass tubes. The eluent is then concentrated to $2 \mathrm{~mL}$ under nitrogen. In order to get rid of the deposited impurities, the analyte is transferred into a $10-\mathrm{mL}$ glass vial. $800 \mu \mathrm{L}$ of methanol is used to rinse the graduated glass tubes for the complete transfer of the analytes and $1200 \mu \mathrm{L}$ of $0.01 \%$ aqueous ammonium hydroxide solution is added. To reduce matrix interferences and ensure the analyte's concentrations in the quantitative range of LC-QTOF-MS, sewage sludge extracts are further diluted to $5 \mathrm{~mL}$ by adding $1 \mathrm{~mL}$ of a 70:30 (v/v) methanol/aqueous ammonium hydroxide $(0.01 \%)$ solution. The extracts are stored at $4{ }^{\circ} \mathrm{C}$ until analysis. A $500-\mu \mathrm{L}$ aliquot of extract is transferred to a $1.5-\mathrm{mL}$ glass auto sampler vial.

\subsection{Analytical procedure}

A sample volume of $10 \mu \mathrm{L}$ is injected into the system of HPLC-QTOF-MS. After the base-lined separation by the C18 reversed column, PFOS and PFOA compounds are identified and further quantitatively analyzed by single mass mode using characteristic ions of $[\mathrm{M}-\mathrm{K}]^{-}$at $m / z 498.93$ and $[\mathrm{M}-\mathrm{COOH}]^{-}$ at $m / z 368.97$ for the determination of PFOS and PFOA, respectively. Quantitative calculation is performed based on the ratio of the peak areas of the analytes to that of the standards. Three analysis replicates are performed for all the standards and samples.

In order to obtain the calibration curves of PFOS and PFOA, the matrix-matched calibration graphs for PFOS and PFOA are prepared by injecting extracted blank spiked with increasing amounts of standard ranging from 0 to $200 \mathrm{ng} / \mathrm{g}$. The matrix-matched standards are then analyzed by the system of HPLC-QTOF-MS and the curves are drawn based on the average peak areas of PFOS and PFOA, which give an acceptable linearity $\left(\gamma^{2}>0.998\right)$ over the test range.

For the confirmation of the feasibility of the proposed method in practical analysis of the sewage sludge samples, spiked recovery experiments are carried out by adding three levels of PFOS
$(5,80,125 \mathrm{ng} / \mathrm{g})$ and PFOA $(8,80,160 \mathrm{ng} / \mathrm{g})$ into a blank sewage sludge from Haikou and processed the pretreatment steps as described above, finally analyzed by HPLC-QTOF-MS. Five replicates are carried out to evaluate the precision of the analytical procedure.

\section{Results and discussion}

\subsection{Optimization of buffer levels in mobile phase}

According to previous reports, the volatile buffer ammonium acetate at pH 6.0 is found the optimum buffer for the ionization of PFOA and PFOS [18,19]. Additionally, the concentrations of the buffer may affect the intensity of signal to noise $(\mathrm{S} / \mathrm{N})$ ratio of the tested chemicals. Effects of concentration on the intensity of signal to noise $(\mathrm{S} / \mathrm{N})$ ratio are studied by adding different concentrations of ammonium acetate $(0,1,5$ and $10 \mathrm{mmol} / \mathrm{L})$ to the inorganic phase. With the increase of the concentration of buffer, the intensities of PFOS and PFOA are enhanced. But $10 \mathrm{mmol} / \mathrm{L}$ ammonium acetate leads to less intensity both of PFOS and PFOA (data not shown). The highest $\mathrm{S} / \mathrm{N}$ ratio of PFOS and PFOA are obtained using $5 \mathrm{mM}$ ammonium acetate in water-methanol. The optimized mobile phase composition is applied to the following experiments.

\subsection{Optimization of $Q T O F-M S$ parameters}

The impacts of mass spectrometric parameters, such as cone, capillary and collision voltage, are investigated by the analysis of standard solution of PFOS $(10 \mathrm{ng} / \mathrm{mL})$ and PFOA $(10 \mathrm{ng} / \mathrm{mL})$. It is found that the intensity of PFOS and PFOA varies with the increasing of cone voltage from 20 to $60 \mathrm{~V}$. With the increase of cone voltage, the response of PFOS and PFOA increase initially, and then they reach the maximum, followed by a fall trend. The curves of ion intensity versus cone voltage are obtained. As shown in Fig. 1, a sample cone voltage of $35 \mathrm{~V}$ is finally selected for further experiments. Under these experimental conditions, single MS spectra of PFCs obtained with the QTOF-MS instrument agree with those previously reported using quadrupole and ion trap mass spectrometry [21].

Capillary voltage is another important parameter greatly affecting the sensitivities. The responses of PFOS and PFOA significantly increase with capillary voltage varying from 1.0 to $2.5 \mathrm{kV}$, and then obtain stable state. As we all know, at relatively high voltages, the tip of the capillary can discharge, which would damage the ion source. So a $2.5 \mathrm{kV}$ of capillary voltage is ultimately chosen.

With the enhancement of collision energy, the ratios of the intensities of specific fragment ions to those of the precursor ions increased significantly, whereas it is essential to maintain enough intensity of precursor ions to confirm the existence of PFOS and PFOA. In the proposed method, MS/MS mode is applied to confirm the feasibility and veracity of single mode. $35 \mathrm{~V}$ collision voltages are selected to produce specific fragmentation ions. In the determination of PFOS and PFOA in sewage sludge samples, single ms mode is used to confirm and calculate them. Individual analyte standard is directly infused to tune the 


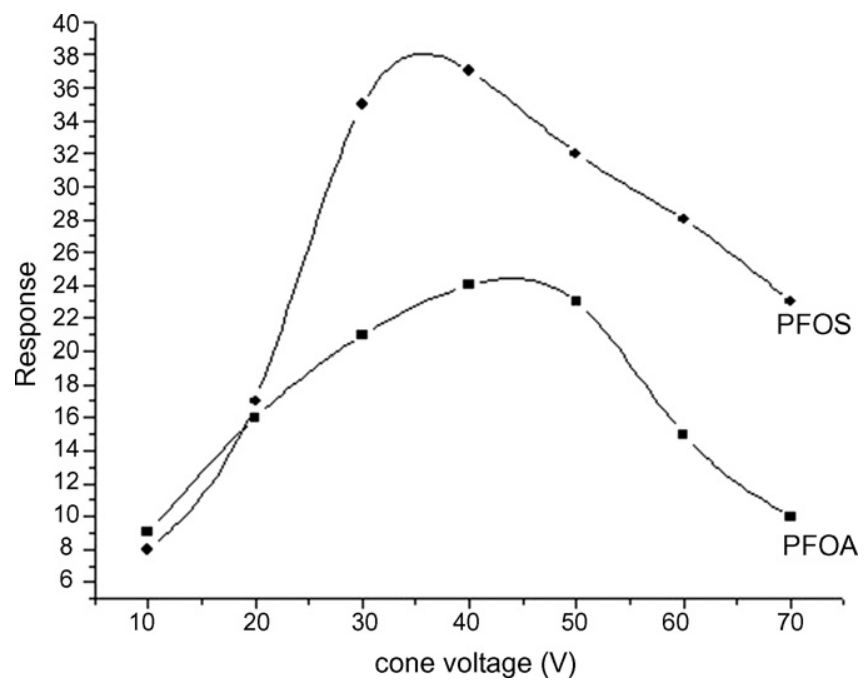

Fig. 1. The effect of sample cone voltage on the intensity of PFOS and PFOA $(10 \mathrm{ng} / \mathrm{mL}$ for PFOS, $10 \mathrm{ng} / \mathrm{mL}$ for PFOA), flow rate is $10 \mu \mathrm{L} / \mathrm{min}$; capillary voltage is $2.5 \mathrm{kV}$; collusion energy is $5 \mathrm{~V}$; source and desolvation temperatures, 120 and $250{ }^{\circ} \mathrm{C}$; cone and desolvation gas flows (nitrogen), 100 and $650 \mathrm{~L} / \mathrm{h}$, respectively.

instrument based on the parent ion and to determine the fragment ions of interest. All the optimal values achieved are used for the following analysis of the standards and the sewage sludge samples.

\subsection{Fragmentation pathways of the PFOS and PFOA}

Previous studies using triple quadrupole mass spectrometry have elucidated the structure of PFCs [20]. To obtain a more accurate assignment, the MS/MS spectra of PFOS and PFOA using the QTOF instrument are studied. Collision energies for all analytes are set at $35 \mathrm{~V}$ in order to obtain both the maximum signals for product ions and at least $\sim 10 \%$ for the parent ion (optimal values are given in Section 3). In general, the MS/MS spectra of PFOS and PFOA are similar to those obtained previously with triple quadrupole mass spectrometry in terms of fragmentation patterns [20]. However, high-resolution makes it obtain more accurate mass. Fig. 2 shows the MS/MS spectrum obtained with the QTOF-MS instrument for PFOS, where the assignments with their corresponding mass accuracies are also indicated. For PFOS, the fragment at $m / z, 79.95$ and 98.95 correspond to the $\mathrm{SO}_{3}{ }^{-}$and $\mathrm{FSO}_{3}{ }^{-}$, respectively [10]. For PFOA (Fig. 3), $\mathrm{m} / \mathrm{z}$ 368.97, 168.98 and 118.99 correspond to the $\mathrm{C}_{7} \mathrm{~F}_{15}{ }^{-}, \mathrm{C}_{3} \mathrm{~F}_{7}{ }^{-}$and $\mathrm{C}_{2} \mathrm{~F}_{5}{ }^{-}$, respectively.

\subsection{Method validation}

Linear response range and the limits of quantitation of PFOS and PFOA using the LC-QTOF-MS system are studied to evaluate the feasibility and veracity of the proposed method in the application to analyzing sewage sludge sample. For sewage sludge samples, matrix-related ion interference may exist using LC-MS/MS analysis [20], which leads to the decrease in detector response. Using a matrix-matched standard can compensated for the interference. The analytical characteristics of the method,

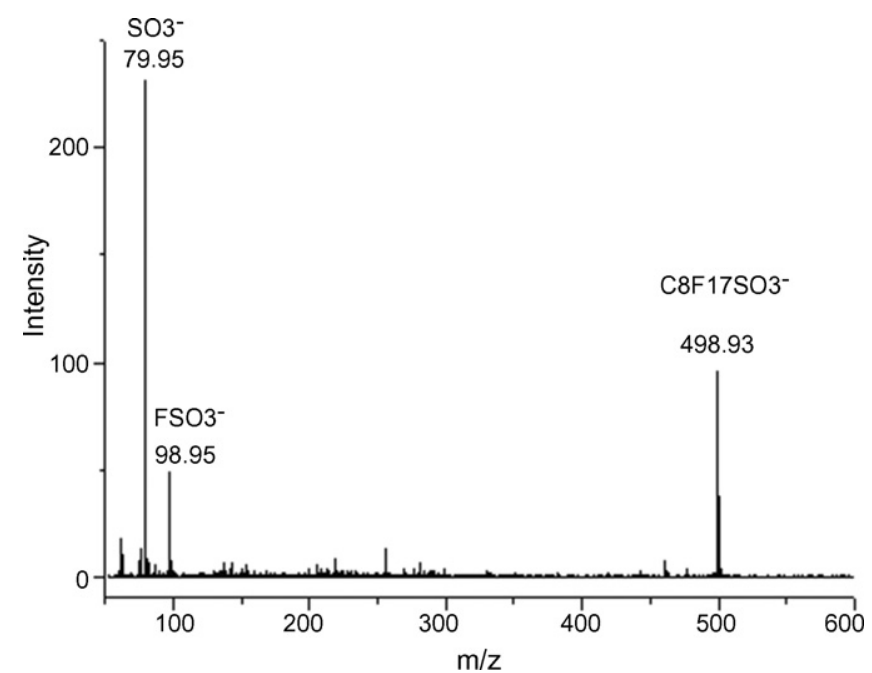

Fig. 2. The fragmentation ions of PFOS. The concentration of PFOS is $100 \mathrm{ng} / \mathrm{mL}$; flow rate is $10 \mu \mathrm{L} / \mathrm{min}$. Capillary voltage and sample cone: $2.5 \mathrm{kV}$ and $35 \mathrm{~V}$; collusion energy is $35 \mathrm{~V}$; source and desolvation temperatures: 120 and $250^{\circ} \mathrm{C}$; cone and desolvation gas flows (nitrogen): 100 and $650 \mathrm{~L} / \mathrm{h}$, respectively. A window width of $\pm 0.05 \mathrm{Da}$ is selected.

such as linear response range, reproducibility and limits of quantitation are investigated and the results depicted in Table 1. The linearities of the PFOS and PFOA are calculated from the fivelevel calibration curve over the range from 5 to $125 \mathrm{ng} / \mathrm{g}$ for PFOS and 8 to $160 \mathrm{ng} / \mathrm{g}$ for PFOA, and an acceptable linearity $\left(\gamma^{2}=0.998\right)$ in the test ranges are obtained. Quantification is based on the response of the external standards that bracketed the concentrations found in samples.

The limits of detection (LOD) based on three times of S/N ratio are 1.5 and $2.4 \mathrm{ng} / \mathrm{g}$ for PFOS and PFOA, separately. The limits of quantitation (LOQ), defined as $\mathrm{S} / \mathrm{N}$ ratio of 10 are 5 and $8 \mathrm{ng} / \mathrm{g}$ for PFOS and PFOA in sewage sludge samples, respectively.

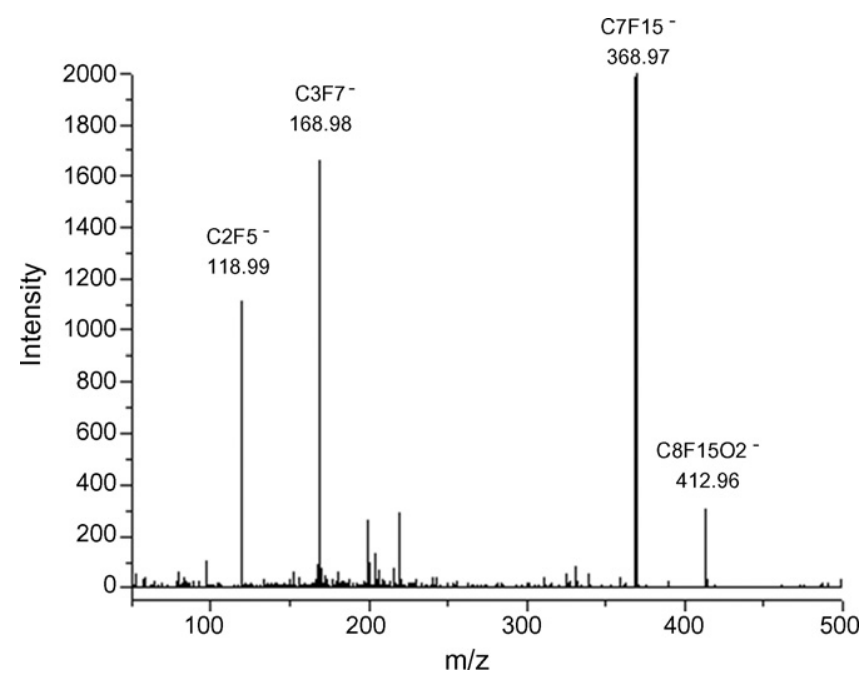

Fig. 3. The fragmentation ions of PFOA. The concentration of PFOA is $500 \mathrm{ng} / \mathrm{mL}$; flow rate is $10 \mu \mathrm{L} / \mathrm{min}$. Capillary voltage and sample cone: $2.5 \mathrm{kV}$ and $35 \mathrm{~V}$; collusion energy is $35 \mathrm{~V}$; source and desolvation temperatures: 120 and $250^{\circ} \mathrm{C}$; cone and desolvation gas flows (nitrogen): 100 and $650 \mathrm{~L} / \mathrm{h}$, respectively. A window width of $\pm 0.05 \mathrm{Da}$ is selected. 
Table 1

The standard curves of the analytical method

\begin{tabular}{llllll}
\hline Analyte & Quantitation ions & Linear curve & $\gamma^{2}$ & Linear range $(\mathrm{ng} / \mathrm{g})$ & LOD $(\mathrm{ng} / \mathrm{g})$ \\
\hline PFOS & {$[\mathrm{M}-\mathrm{K}]^{-} m / z 498.93$} & $y=0.9834 x+1.0869$ & 0.998 & $5-125$ & 1.5 \\
PFOA & {$[\mathrm{M}-\mathrm{COOH}]^{-} m / z 368.97$} & $y=1.0568 x+1.1667$ & 0.999 & $8-160$ & 5 \\
\hline
\end{tabular}

The accuracy, precision, and stability of the developed method are evaluated by the spiked recovery experiments at low, middle- and high-spiked concentrations of PFOS and PFOA, respectively. The results shown in Table 2 indicate that the spiked recoveries are in the range of $85-114 \%$ for PFOS and $71-98 \%$ for PFOA. The relative standard deviations are less than $15 \%$. The results are in compliance with those reported in the literature [20]. The results reveal that the method developed here could be applied to the analysis of PFOA and PFOS in sewage sludge samples.

\subsection{Application}

The proposed method is successfully used to analyze 16 sewage sludge samples from China. These samples collected from 16 cities, which distribute in large area of China, to some degree, could represent the contamination level of PFOS and PFOA in China. Fig. 4 shows the mass chromatogram comparison of the standards and the sewage sludge sample from Yinchuan. PFOS and PFOA are confirmed by comparing the mass spectrum and the corresponding retention time of samples with those of the standards. The concentrations are estimated by external standard method based on the matrix-matched standard calibration curves. Table 3 shows the contamination levels of PFOS and PFOA in sewage sludge samples. It could be found that PFOS and PFOA compounds are detected in most of the sewage sludge samples and the concentrations range from 278 to $5383 \mathrm{ng} / \mathrm{g}$ (dry weight), which is generally higher than the levels reported previously [20]. According to Table 3 , it could be found that the contamination of PFOS and PFOA spreads widely in China, though there are no large production plants for PFCs. The values of PFOS and PFOA detected in Xiamen1 and Xiamen2 differ from each other greatly, it is might due to different waste water sources. According to previous reports, the waste water from industry plants, the concentrations of PFCs are high, while from domestic products, the concentrations of PFCs are low. The current contamination might come from the release

Table 2

Spiked recoveries of PFOS and PFOA in a Haikou sewage sludge sample $(n=5)$

\begin{tabular}{lcc}
\hline Analyte & Spiked level $(\mathrm{ng} / \mathrm{g})$ & Mean recovery \pm R.S.D. $(\%)$ \\
\hline PFOS & 5 & $99 \pm 15$ \\
& 80 & $96 \pm 11$ \\
& 125 & $105 \pm 9$ \\
PFOA & 8 & $83 \pm 12$ \\
& 80 & $88 \pm 10$ \\
& 160 & $84 \pm 8$
\end{tabular}

${ }^{a}$ The Haikou sewage sludge sample served as the blank matrix. The experimental conditions are same as those in Fig. 4 of PFC-containing products. The volatile precursors of PFOS and PFOA might be another potential source for the widespread contamination. PFOS may be present as unreacted residual in consumer products such as clothing and surface-treatment products. Previous study also suggests PFOS can be come from the biotransformation of $N$-ethyl- $N$-(2-hydroxyethyl) perfluorooctanesulfonamide [20]. PFOA, as the precursor of many surface materials, might remain in the product and be released when
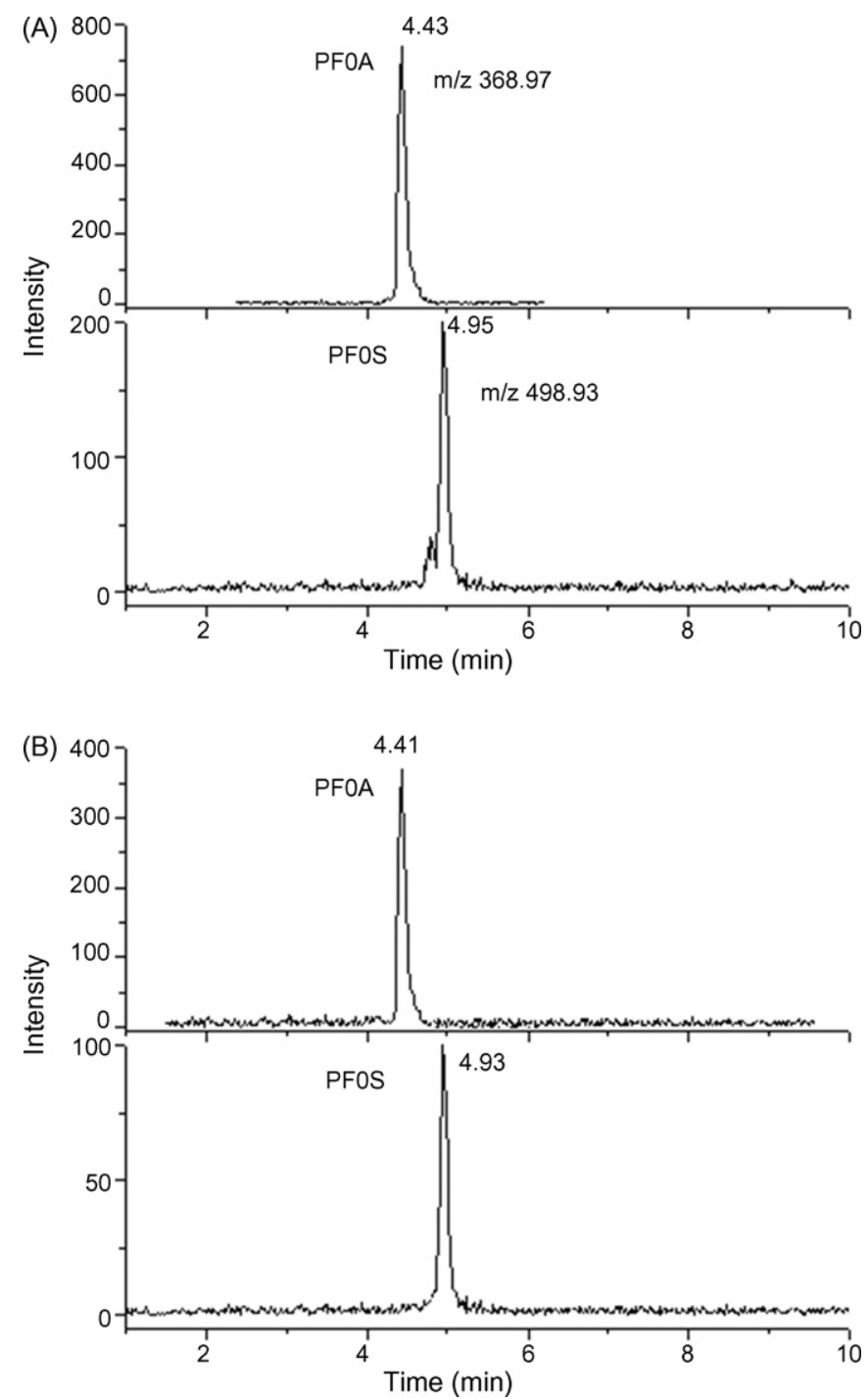

Fig. 4. The comparison of LC-MS ion chromatogram of PFOS and PFOA in standard mixture ( $45 \mathrm{ng} / \mathrm{mL}$ for PFOS, $100 \mathrm{ng} / \mathrm{mL}$ for PFOA) with a sample from Yinchuan (PFOS $2560 \mathrm{ng} / \mathrm{g}$, PFOA $3259 \mathrm{ng} / \mathrm{g}$ ). The condition of mass spectrometry is same as those in Fig. 2 except the collusion energy is $5 \mathrm{~V}$. The condition of separation is same with mentioned in Section 2.2, the injection volume is $10 \mu \mathrm{L}$. (A) The chromatogram of standard. (B) The chromatogram of sample. 
Table 3

Concentrations of PFOS and PFOA (ng/g dry weight) in sewage sludge samples (average concentration \pm standard error) $(n=3)$

\begin{tabular}{lll}
\hline Sample site & PFOS & PFOA \\
\hline Baoding & N.D. & N.D. \\
Beixiaohe & $280 \pm 34$ & $492 \pm 69$ \\
Guangzhou1 & $5383 \pm 108$ & $4591 \pm 89$ \\
Guangzhou2 & $1015 \pm 51$ & $1680 \pm 118$ \\
Haerbin & N.D. & $1650 \pm 82$ \\
Haikou & N.D. & N.D. \\
Hangzhou & N.D. & N.D. \\
Hefei & $236 \pm 19$ & $493 \pm 49$ \\
Linyi & N.D. & N.D. \\
Liuzhou & $2522 \pm 76$ & $1792 \pm 90$ \\
Shanghai & $472 \pm 52$ & $454 \pm 54$ \\
Shenyang & $973 \pm 78$ & $2159 \pm 86$ \\
Wulumuqi & $1081 \pm 65$ & $4780 \pm 143$ \\
Xiamen1 & $4257 \pm 85$ & $2365 \pm 95$ \\
Xiamen2 & $278 \pm 25$ & $563 \pm 62$ \\
Yinchuan & $2560 \pm 77$ & $3259 \pm 75$ \\
\hline
\end{tabular}

Note $:$ N.D. $=$ not detected. The experimental conditions are same as those in Fig. 4.

overheated. Other potential sources might include industrial sources, consumer products containing residuals, and releases of PFC-containing aqueous film-forming foam (AFFF) from firefighting activity and training, etc. It still needs further investigations on the potential sources of the PFOS and PFOA in China. There is not enough toxicological data affirming the safe levels of PFOA and PFOS for environmental organisms and human health. It should cause more concerns on the occurrence of these compounds in environment and organisms. More researches should be carried out for the further risk assessment of environmental contamination of PFCs.

\section{Conclusion}

LC-QTOF-MS is a powerful tool of yields full-scan mass spectrum about structure of the molecule to enable unequivocal confirmation of the presence of the PFOS and PFOA. The method using C18-SPE for sample pretreatment and LC-QTOFMS system for the analysis of PFOS and PFOA in sewage sludge samples is firstly developed herein. The satisfying spiked recoveries and R.S.D.s confirm the reliability of the proposed method. The acceptable sensitivities endue it practical application to analyze PFOS and PFOA contamination in sewage sludge samples.

\section{Acknowledgement}

This research is supported by a grant from the Chinese Academy Sciences no. KZCX3-SW-431.

\section{References}

[1] 3M, Health and Environmental Assessment of Perfluorooctane Sulfonic Acid and its Salts, EPA docket AR-226-1486, US Environmental Protection Agency, Washington, DC, 2003

[2] M.M. Schultz, D. Barofsky, J.A. Field, Environ. Sci. Technol. 38 (2004) 1828.

[3] G.W. Olsen, T.R. Church, J.P. Miller, J.M. Burris, K.J. Hansen, J.K. Lundberg, J.B. Armitage, R.M. Herron, Z. Medhdizadehkashi, J.B. Nobiletti, E.M. O'Neill, J.H. Mandel, L.R. Zobel, Environ. Health Perspect. 111 (2003) 1892.

[4] J.P. Giesy, K. Kannan, Environ. Sci. Technol. 35 (2001) 1339.

[5] J.P. Giesy, K. Kannan, Environ. Sci. Technol. 36 (2002) 147A.

[6] K. Kannan, J. Koistinen, K. Beckmen, T. Evans, J. Garzelany, K.J. Hansen, P.D. Jones, J.P. Giesy, Environ. Sci. Technol. 35 (2001) 1593.

[7] K. Kannan, S.P. Hansen, C.J. Franson, W.W. Bowerman, K.J. Hansen, P.D. Jones, J.P. Giesy, Environ. Sci. Technol. 35 (2001) 3065.

[8] K. Kannan, J.L. Newsted, R.S. Halbrook, J.P. Giesy, Environ. Sci. Technol. 36 (2002) 2566

[9] K. Kannan, S. Corsolini, J. Falandysz, G. Oehme, S. Focardi, J.P. Giesy, Environ. Sci. Technol. 36 (2002) 3210.

[10] K. Kannan, J.W. Choi, N. Iseki, K. Senthilkumar, D.H. Kim, S. Masunaga, J.P. Giesy, Chemosphere 49 (2002) 225.

[11] C. Lau, J.L. Butenhoff, J.M. Rogers, Toxicol. Appl. Pharmacol. 198 (2004) 231

[12] M.K. So, S. Taniyasu, N. Yamashita, J.P. Giesy, J. Zheng, Z. Fang, S.H. Im, P.K.S. Lam, Environ. Sci. Technol. 38 (2004) 4056.

[13] C.L. Tseng, L.L. Liu, C.M. Chen, W.H. Ding, J. Chromatogr. A 1105 (2006) 119.

[14] A. Karrman, B. van Bavel, U. Jarnberg, L. Hardell, G. Lindstrom, Anal. Chem. 77 (2005) 864.

[15] B. Boulanger, J. Vargo, J.L. Schnoor, K.C. Hornbuckle, Environ. Sci. Technol. 38 (2004) 4064

[16] E. Barceľ̌o-Barrachina, E. Moyano, M.T. Galceran, J. Chromatogr. A 1054 (2004) 409.

[17] X. Fang, X. Fan, Y. Tang, J. Chen, J. Lu, J. Chromatogr. A 1036 (2004) 233

[18] C.A. Moody, W.C. Kwan, J.W. Martin, D.C.G. Muir, S.A. Mabury, Anal. Chem. 73 (2001) 2200.

[19] J.W. Martin, S.A. Mabury, K. Kannan, U. Berger, P. de Voogt, J. Field, J. Franklin, J.P. Giesy, T. Harner, D.C.G. Muir, B. Scott, M. Kaiser, U. Järnberg, K.C. Jones, H. Schroeder, M. Simcik, C. Sottani, B. Van Bavel, A. Kärrman, G. Lindström, S. van Leeuwen, Environ. Sci. Technol. 37 (2004) 248A.

[20] C.P. Higgins, J.A. Field, C.S. Criddle, R.G. Luthy, Environ. Sci. Technol. 39 (2005) 3946

[21] C.Li. Tseng, L.L. Liu, C.M. Chen, W.H. Ding, J. Chromatogr. A 1105 (2006) 119 